\title{
Dynamics of extracellular matrix in ovarian follicles and corpora lutea of mice
}

\author{
Helen F. Irving-Rodgers • Katja Hummitzsch • Lydia S. Murdiyarso • \\ Wendy M. Bonner • Yoshikazu Sado • Yoshifumi Ninomiya • John R. Couchman • \\ Lydia M. Sorokin • Raymond J. Rodgers
}

Received: 20 June 2009 / Accepted: 2 November 2009/Published online: 23 December 2009

(C) The Author(s) 2009. This article is published with open access at Springerlink.com

\begin{abstract}
Despite the mouse being an important laboratory species, little is known about changes in its extracellular matrix (ECM) during follicle and corpora lutea formation and regression. Follicle development was induced in mice (29 days of age/experimental day 0) by injections of pregnant mare's serum gonadotrophin on days 0 and 1 and ovulation was induced by injection of human chorionic
\end{abstract}

This research was supported by the National Health and Medical Research Council of Australia, a Colin Matthews Research Grant from the Research Centre for Reproductive Health, The University of Adelaide, the Clive and Vera Ramaciotti Foundation, NIH grant AR36457 and The Danish National Research Foundation.

H. F. Irving-Rodgers $\cdot$ K. Hummitzsch $\cdot$ L. S. Murdiyarso •

W. M. Bonner $\cdot$ R. J. Rodgers $(\bowtie)$

Research Centre for Reproductive Health,

Discipline of Obstetrics and Gynaecology, School of Paediatrics

and Reproductive Health, Robinson Institute,

The University of Adelaide,

Adelaide, SA 5005, Australia

e-mail: ray.rodgers@adelaide.edu.au

Y. Sado

Division of Immunology, Shigei Medical Research Institute,

Okayama 701, Japan

\section{Y. Ninomiya}

Department of Molecular Biology and Biochemistry,

Okayama University Medical School,

Okayama 700, Japan

\section{J. R. Couchman}

Biomedicinsk Institute, Biocenter, University of Copenhagen,

Ole Maoløes Vej 5,

2200 Copenhagen N, Denmark

\section{M. Sorokin}

Institute for Physiological Chemistry and Pathobiochemistry,

Münster University,

Waldeyerstrasse 15 ,

48149 Münster, Germany gonadotrophin on day 2. Ovaries were collected for immunohistochemistry ( $n=10$ per group) on days 0,2 and 5 . Another group was mated and ovaries were examined on day $11(n=7)$. Collagen type IV $\alpha 1$ and $\alpha 2$, laminin $\alpha 1, \beta 1$ and $\gamma 1$ chains, nidogens 1 and 2 and perlecan were present in the follicular basal lamina of all developmental stages. Collagen type XVIII was only found in basal lamina of primordial, primary and some preantral follicles, whereas laminin $\alpha 2$ was only detected in some preantral and antral follicles. The focimatrix, a specialised matrix of the membrana granulosa, contained collagen type IV $\alpha 1$ and $\alpha 2$, laminin $\alpha 1, \beta 1$ and $\gamma 1$ chains, nidogens 1 and 2, perlecan and collagen type XVIII. In the corpora lutea, staining was restricted to capillary sub-endothelial basal laminas containing collagen type IV $\alpha 1$ and $\alpha 2$, laminin $\alpha 1, \beta 1$ and $\gamma 1$ chains, nidogens 1 and 2 , perlecan and collagen type XVIII. Laminins $\alpha 4$ and $\alpha 5$ were not immunolocalised to any structure in the mouse ovary. The ECM composition of the mouse ovary has similarities to, but also major differences from, other species with respect to nidogens 1 and 2 and perlecan.

Keywords Follicle - Corpus luteum - Extracellular matrix . Collagen $\cdot$ Laminin $\cdot$ Perlecan $\cdot$ Nidogen $\cdot$

Mouse (CBAxC57BL/6F1)

\section{Introduction}

Basal laminas are specialised sheets of extracellular matrix (ECM; Paulsson 1992; Timpl and Brown 1996) that underlie epithelial and endothelial cells or envelop whole cells such as nerve or muscle cells. They separate cells from adjoining stroma, influence their behaviour and can selectively retard the passage of cells and molecules 
(Paulsson 1992; Timpl and Brown 1996). Basal laminas are composed of lattice-type networks of collagen type IV and laminin, which are stabilised by the binding of entactin/ nidogen 1 or 2 (Schymeinsky et al. 2002) and probably also of fibulins and heparan sulphate proteoglycans, such as perlecan, to the collagen and laminin. Collagen type IV comprises three $\alpha$ chains of which there are six different chains of collagen type IV $\alpha(\alpha 1-\alpha 6$, each encoded by a separate gene; Hay 1991) and various combinations of collagen type IV $\alpha$ chains can assemble to form unique isoforms in vivo (e.g. $\alpha 1 \alpha 1 \alpha 2, \alpha 3 \alpha 4 \alpha 5$; Sado et al. 1998). Laminins are composed of three different chains, viz. $\alpha, \beta$ and $\gamma$ chains (Aumailley et al. 2005), of which there are five different $\alpha$ chains, three $\beta$ chains and three $\gamma$ chains (all encoded by separate genes). The combination of $\alpha, \beta$ and $\gamma$ chains potentially gives rise to $45(5 \times 3 \times 3)$ different molecular combinations, with alternative splicing resulting in more combinations (Aumailley et al. 2005). Collagen type XVIII, which has structural properties of both a collagen and proteoglycan, is also associated with the basal laminas, in particular the sub-endothelial basal laminas (Marneros and Olsen 2005). Basal laminas thus can vary considerably in composition, which might influence their physical and biological properties and have differential effects on cells.

In adult ovaries, considerable tissue remodelling occurs as follicles grow, ovulate or regress and when corpora lutea are formed or regress. These processes require the expansion of basal laminas within follicles and corpora lutea and their later degradation. Much of the research on basal lamina matrices has been conducted in bovine (for a review, see Irving-Rodgers et al. 2006c), with fewer studies in humans (Irving-Rodgers et al. 2006b, 2009b). Most of the focus in follicles has been on the follicular basal lamina underlying the membrana granulosa, the changes that it undergoes during follicular development and the two ultrastructural phenotypes that are related to granulosa cell shape (Irving-Rodgers and Rodgers 2000) and to oocyte quality (Irving-Rodgers et al. 2009b). The composition of the sub-endothelial basal lamina in the theca has also been documented (for a review, see Rodgers et al. 2003) and a novel type of basal lamina matrix, the focimatrix (focal intra-epithelial matrix), has been identified (Irving-Rodgers et al. 2004). The focimatrix appears about 10 days before ovulation in the bovine. It is deposited as plaques or aggregates of basal lamina material between the epithelial granulosa cells in follicles. It first appears in follicles greater than $5 \mathrm{~mm}$ in diameter in bovine, and its abundance increases as the follicles enlarge. Although discovered in the cow, the focimatrix also occurs in sheep (Huet et al. 1997), humans (Yamada et al. 1999; Alexopoulos et al. 2000), mice (Nakano et al. 2007), rats (our interpretation of data from Frojdman et al. [1998]) and pigs (unpublished observations). The novel aspect of this matrix is its conformation. By wrapping around cells or groups of cells, basal laminas make compartments within tissues. The focimatrix, however, does not form a continuous layer and thus presumably does not perform known basal lamina functions. In the corpora lutea, the basal lamina matrix is largely confined to the sub-endothelial matrix and arteriolar smooth muscle cells but laminins constituting a parenchymal matrix have also been identified (Irving-Rodgers et al. 2004, 2006c; Nakano et al. 2007).

The goals in this area of research are now to discover the roles of the dynamic changes in the matrix. Recently, the expression of a number of focimatrix genes in granulosa cells has been shown to be highly correlated with the expression of CYP11A1 (cholesterol side-chain cleavage) and CYP19A1 (aromatase; Irving-Rodgers et al. 2009a). Additionally, dominant follicles have elevated levels of both CYP11A1 mRNA and of focimatrix (quantitated by using antisera to laminin 111) independently of size differences between dominant and subordinate follicles (Irving-Rodgers et al. 2009a). On the basis of such findings, the focimatrix has been suggested to induce the expression of steroidogenic enzymes as a mechanism for inducing the dominance of one follicle over another (Irving-Rodgers et al. 2009a). Furthermore, the importance of the extracellular milieu has been emphasised by the finding that isolated follicles cultured in polymerised alginate hydrogels grow exceptionally well (Kreeger et al. 2005; Xu et al. 2009).

Mice are commonly used as a model of choice for producing transgenic animals and for genetic N-ethyl-N-nitrosourea mutagenesis screening (Papathanasiou and Goodnow 2005). As such, we need to increase our knowledge of the ovarian matrix in the murine ovary. Currently, much of the study of the matrix in mouse ovaries has focused on the cumulus oocyte complex (Camaioni et al. 1996; Rodgers et al. 2003; Dunning et al. 2007) or stromal matrix. However, studies have identified collagen type IV isotypes (Frojdman et al. 1998; Nakano et al. 2007) and the glycoprotein usherin, which contains four domains common to basal laminas and ECM (thrombospondin, laminin type IV, laminin epidermalgrowth-factor-like and fibronectin type III) (Pearsall et al. 2002) in the murine ovary. Here, we expand our knowledge on the composition of basal lamina matrices in the murine ovary, in both non-pregnant and pregnant mice.

\section{Materials and methods}

\section{Experimental animals}

All animal experimental protocols were approved by both The University of Adelaide and The Institute of Medical \& Veterinary Science (IMVS) Animal Ethics Committees and were conducted in accordance with the National Research Council (NRC) publication Guide for Care and Use of 
Laboratory Animals (copyright 1996, National Academy of Science). Twenty 5-day-old female $\mathrm{CBA} \times \mathrm{C} 57 \mathrm{BL} / 6 \mathrm{~F} 1$ mice in the weight range of $10-12 \mathrm{~g}$ were obtained from the Laboratory Animal Services, The University of Adelaide, and housed in the IMVS Animal Care Facility with controlled light cycles (12 h light:12 h dark). Four days later, the mice were subjected to induction of ovulation with gonadotrophins. Pregnant mare's serum gonadotrophin (PMSG; Intervet, Victoria, Australia) was administered at 5 IU intraperitoneally on days 0 and 1 and 5 IU human chorionic gonadotrophin (hCG; Organon, NSW, Australia) on day 2. Some groups were mated by caging females with 7-week-old fertile CBA males overnight ( 2 females with 1 male per cage). Vaginal plugs were checked $24 \mathrm{~h}$ later and the time that vaginal plugs appeared was noted. Mice $(n=10$ per group) were killed with carbon dioxide on days $0,2,5$ and 11 relative to the first injection of PMSG. Both ovaries from each animal were harvested, one ovary being fixed in $4 \%$ paraformaldehyde (Merck, Victoria, Australia) in $0.1 \mathrm{M}$ phosphate buffer ( $\mathrm{pH}$ 7.4). These tissues were then washed several times in phosphate-buffered saline (PBS) and dehydrated in ascending series of ethanol from $70 \%$ to 100\% (Leica TP1020, Automatic Tissue Processor; Leica, Nussloch, Germany) and embedded in paraffin blocks (Leica EG1140H, Paraffin Embedding Station) for subsequent haematoxylin-eosin staining. The other ovary from each animal was frozen, embedded in Optimal Cutting Temperature compound (Sakura Finetechnical, Tokyo, Japan) and stored at $-80^{\circ} \mathrm{C}$ for subsequent immunohistochemistry. The numbers of fetuses in the pregnant animals were recorded. Additional tissues were collected from these animals and served as positive controls for immunostaining when no staining was detected in the ovaries.

\section{Histology}

Paraffin sections $(3 \mu \mathrm{m})$ were cut by using a CM1850 V2.2 Leica microtome (Leica Microsystems, Nussloch, Germany) and collected on Superfrost glass slides (HD Scientific, Australia). After $1 \mathrm{~h}$ incubation at $60^{\circ} \mathrm{C}$, the slides were dewaxed in xylene $(2 \times 5 \mathrm{~min})$ and in $100 \%$ ethanol $(2 \times 5 \mathrm{~min})$ and rinsed in distilled water. The sections were stained with haematoxylin (catalogue no. GHS232; Sigma) for $5 \mathrm{~min}$, rinsed in tap water and in acid alcohol for $5 \mathrm{~s}$ and subsequently stained in eosin (catalogue no. HT110132; Sigma) for $20 \mathrm{~s}$. Afterwards, the slides were rinsed in tap water, in $70 \%$ ethanol for $1 \mathrm{~min}$, in $100 \%$ ethanol $(3 \times 1 \mathrm{~min})$ and in xylene $(3 \times 5 \mathrm{~min})$ and mounted in Pertex (Medite, Burgdorf, Germany). The haematoxylineosin-stained sections were used for the classification of atresia, the estimation of average numbers of healthy antral follicles and corpora lutea on days $0,2,5$ and 11 and the counting of implantation sites in the mated mice.
Immunohistochemistry

Table 1 summarises the antibodies used for immunofluorescence and the relevant fixation conditions. Localisation was undertaken by using an indirect immunofluorescence method (Irving-Rodgers et al. 2002). Tissue sections $(7 \mu \mathrm{m})$ were cut on a CM1800 Leica cryostat (Adeal, Altona North, Vic, Australia), collected on superfrost glass slides and stored at $-20^{\circ} \mathrm{C}$ until use. Unfixed sections were dried under vacuum for $5 \mathrm{~min}$ followed either by fixation in $100 \%$ acetone, $10 \%$ neutral buffered formalin (BFS), $100 \%$ ethanol or left unfixed. Sections were then rinsed $(3 \times 5 \mathrm{~min})$ in changes of hypertonic PBS $(10 \mathrm{mM}$ sodium/potassium phosphate with $0.274 \mathrm{M} \mathrm{NaCl}, 5 \mathrm{mM} \mathrm{KCl}, \mathrm{pH}$ 7.2) before treatment with blocking solution $(10 \%$ normal donkey serum [catalogue no. D-9663; Sigma] in antibody diluent containing $0.55 \mathrm{M}$ sodium chloride and $10 \mathrm{mM}$ sodium phosphate, $\mathrm{pH}$ 7.1) for $30 \mathrm{~min}$ at room temperature. Incubation with primary antibodies was carried out overnight at room temperature. The secondary antibodies used and the labelling conditions are summarised in Table 1. For dual localisations, sections were also treated with the nuclear stain 4',6'-diamidino-2-phenylindole dihydrochloride (DAPI) solution (Molecular Probes, Eugene, Ore., USA). Sections were mounted in mounting medium for fluorescence (catalogue no. S3023; Dako, Carpinteria, Calif., USA) and photographed with an Olympus BX50 microscope with an epifluorescence attachment and a Spot RT digital camera (Diagnostic Instruments, Sterling Heights, Mich., USA).

\section{Results}

Ovaries were examined at 29 days of age (day 0) before the onset of puberty and on day 2 after induction of follicle growth with PMSG (injections days 0 and 1). Ovulation was induced with hCG (day 2). One group of mice were not mated and their ovaries were examined on day 5, whereas the other group was mated and ovaries were examined on day 11 relative to first day of injection of PMSG (day 0). The ovaries at day 0 exhibited small antral follicles (between 200 and $400 \mu \mathrm{m}$ ); at day 2, they contained additional larger antral follicles (between 300 and $550 \mu \mathrm{m}$ ); on days 5 and 11 , the ovaries also contained corpora lutea. The percentages of healthy antral follicles were (mean \pm SEM): $51 \pm 8 \%, 87 \pm 4 \%, 51 \pm 10 \%$ and $62 \pm 7 \%$ on days $0,2,5$ and 11 , respectively. Day-5 animals $(n=10)$ had $6 \pm 1$ corpora lutea per ovary cross section. Fifteen animals examined following ovulation induction and mating were pregnant $(75 \%)$ with $14 \pm 2$ implantation sites per animal at day 11 . Only the pregnant animals from this group were examined further. Table 2 shows the number of 
Table 1 Primary antibodies, secondary antibodies, and labelling and fixation conditions used for immunohistochemistry for each antigen. Secondary antibodies used were either biotin-SP-conjugated AffiniPure donkey anti-rat IgG (BD anti-rat, 1:100; catalogue no. 712-066153) or anti-rabbit IgG (BD anti-rbt, 1:100; catalogue no. 711-066-152), followed by Cy3-conjugated streptavidin (SA-Cy3, 1:100; catalogue no. 016-160-084) or fluorescein (DTAF)-conjugated streptavidin (SA-
DTAF, 1:100; catalogue no. 016-010-084), or Cy3-conjugated AffiniPure donkey anti-rabbit IgG (D anti-rbt-Cy3, 1:100; catalogue no. 711-166-152) or fluorescein isothiocyanate (FITC)-conjugated AffiniPure donkey anti-rabbit IgG (D anti-rbt-FITC, 1:100; catalogue no. 711-015-152); all secondary antibodies and conjugated streptavidins were from Jackson ImmunoResearch Laboratories (West Grove, Pa., USA)

\begin{tabular}{|c|c|c|c|c|c|c|c|}
\hline \multirow[t]{2}{*}{ Antigen (species) } & \multicolumn{4}{|c|}{ Primary antibody } & \multirow{2}{*}{$\begin{array}{l}\text { Secondary } \\
\text { antibody }\end{array}$} & \multirow[t]{2}{*}{ Conjugates } & \multirow[t]{2}{*}{ Fixation } \\
\hline & $\begin{array}{l}\text { Host } \\
\text { species }\end{array}$ & $\begin{array}{l}\text { Code or } \\
\text { clone number }\end{array}$ & Source or Reference & Dilution & & & \\
\hline Laminin $\alpha 1$ (mouse) & Rabbit & 317 & Durbeej et al. (1996) & $1: 1000$ & $\begin{array}{l}\text { D anti-rbt-Cy3 } \\
\text { D anti-rbt-FITC }\end{array}$ & $\begin{array}{l}\text { None } \\
\text { None }\end{array}$ & $\begin{array}{l}\text { Unfixed } \\
\text { Unfixed }\end{array}$ \\
\hline Laminin $\alpha 2$ (mouse) & Rat & $4 \mathrm{H} 8-2$ & Sigma Aldrich & $1: 2000$ & D anti-rbt-Cy3 & None & $100 \%$ acetone \\
\hline Laminin $\alpha 4$ (mouse) & Rabbit & 377 & Sorokin et al. (2000) & $1: 500$ & $\mathrm{BD}$ anti-rat & SA-Cy3 & Unfixed \\
\hline Laminin $\alpha 5$ (mouse) & Rabbit & 405 & Sorokin et al. (2000) & $1: 500$ & $\mathrm{BD}$ anti-rat & SA-Cy3 & Unfixed \\
\hline Laminin $\beta 1$ (mouse) & Rat & LT3 & Millipore & $1: 50$ & D anti-rbt-Cy3 & None & Unfixed \\
\hline Laminin $\gamma 1$ (mouse) & Rat & $3 \mathrm{E} 10$ & Sixt et al. (2001) & $1: 2$ & $\mathrm{BD}$ anti-rbt & SA-DTAF & Unfixed \\
\hline $\begin{array}{l}\text { Type IV collagens } \\
\alpha 1-\alpha 6 \text { (human) }\end{array}$ & Rat & - & Sado et al. (1995) & $1: 100$ & $\mathrm{BD}$ anti-rat & SA-Cy3 & $100 \%$ acetone \\
\hline $\begin{array}{l}\text { Type XVIII collagen } \\
\text { (mouse) }\end{array}$ & Rabbit & 92462 & $\begin{array}{l}\text { Erickson and } \\
\text { Couchman (2001) }\end{array}$ & $1: 200$ & $\begin{array}{l}\text { BD anti-rbt } \\
\text { D anti-rbt-FITC }\end{array}$ & $\begin{array}{l}\text { SA-DTAF } \\
\text { None }\end{array}$ & $\begin{array}{l}100 \% \text { acetone } \\
100 \% \text { acetone }\end{array}$ \\
\hline Nidogen-1 (mouse) & Rabbit & 914 & Dziadek et al. (1985b) & $1: 200$ & D anti-rbt-FITC & None & Unfixed \\
\hline Nidogen-2 (mouse) & Rabbit & 1130 & Papaccio et al. (1998) & $1: 800$ & D anti-rbt-FITC & None & $10 \% \mathrm{BFS}$ \\
\hline Perlecan (mouse) & Rabbit & 906 & Dziadek et al. (1985a) & $1: 100$ & $\begin{array}{l}\text { BD anti-rbt } \\
\text { D anti-rbt-FITC }\end{array}$ & $\begin{array}{l}\text { SA-DTAF } \\
\text { None }\end{array}$ & $\begin{array}{l}\text { Unfixed } \\
\text { Unfixed }\end{array}$ \\
\hline PECAM-1/ CD31 & Rat & 390 & BD Phar-mingen & $1: 500$ & $\mathrm{BD}$ anti-rat & SA-Cy3 & $100 \%$ ethanol \\
\hline
\end{tabular}

ovaries examined at each time point for each ECM molecule, either alone or in combination. The results are presented as a description of changes during follicular and luteal development.

Table 2 Numbers of animals examined at each day of the experiment (day $0=29$ days of age and the day of commencement of the first injection of pregnant mare's serum gonadotrophin) for each extracellular matrix molecule, either by immunostaining alone or in combination with another antibody or with the nuclear stain DAPI

\begin{tabular}{lllll}
\hline Matrix & Day 0 & Day 2 & Day 5 & Day 11 \\
\hline Collagen IV $\alpha 1$ & 1 & 0 & 1 & 0 \\
Collagen IV $\alpha 2$ & 1 & 0 & 1 & 0 \\
Laminin $\alpha 1$ & 5 & 4 & 8 & 5 \\
Laminin $\alpha 2$ & 8 & 9 & 8 & 5 \\
Laminin $\alpha 4$ & 1 & 1 & 1 & 1 \\
Laminin $\alpha 5$ & 1 & 1 & 1 & 1 \\
Laminin $\beta 1$ & 8 & 7 & 8 & 5 \\
Laminin $\gamma 1$ & 5 & 4 & 8 & 3 \\
Nidogen-1 & 4 & 4 & 4 & 3 \\
Nidogen-2 & 3 & 4 & 4 & 2 \\
Perlecan & 8 & 9 & 8 & 5 \\
Collagen type XVIII & 6 & 4 & 5 & 3 \\
\hline
\end{tabular}

\section{Matrix in follicles}

The follicular basal lamina contained collagen type IV $\alpha 1$ and $\alpha 2$ at all stages from primordial to large preovulatory stages (data not shown) confirming previous data (Nakano et al. 2007). Laminin $\alpha 1$ was similarly present at all stages (Fig. 1d, e) but laminin $\alpha 2$ was only detected in some follicles and only at the preantral and antral stages (Fig. 1a, b). Laminin $\alpha 4$ or $\alpha 5$ chains were not detected at any stage (Fig. $2 \mathrm{a} / \mathrm{a}^{\prime}, \mathrm{b} / \mathrm{b}^{\prime}$ ). Laminin $\alpha 4$ was detected in heart and skeletal muscle (Fig. 2c, $\mathrm{c}^{\prime}$ ) and laminin $\alpha 5$ was detected in kidney (Fig. 2d), indicating that the antisera reacted with mouse laminins $\alpha 4$ and $\alpha 5$. Laminin $\beta 1$ (Fig. $1 \mathrm{~g}, \mathrm{~h}, \mathrm{~m}, \mathrm{n}$ ) and $\gamma 1$ chains (Fig. 1d, e) were present at all stages of follicular development. Both nidogens 1 and 2 were also present throughout development (Fig. 1j, k, m, n). Of the heparan sulphate proteoglycans examined, perlecan was present at all stages (Fig. 1a, b), whereas collagen type XVIII was present only in primordial and primary follicles and in some preantral but not in antral follicles (Fig. 1g, h).

Focimatrix was present in the preantral and antral follicles at days $0,2,5$ and 11 and appeared as larger and fewer aggregates (approximately six in cross section) than those observed in the bovine ovary (Irving-Rodgers et al. 2004) or occurred as fibrous structures throughout whole 
membrana granulosa. Nidogens 1 and 2 and perlecan (Figs. 1a, b, 3a, b) showed fibrous patterns of expression, whereas the aggregates contained collagen type IV $\alpha 1$ and $\alpha 2$ (data not shown; as observed previously by Nakano et al. [2007]), laminins $\alpha 1$ (Figs. 1d, 3c), $\beta 1$ (Figs. 1g, h, m, $\mathrm{n}, 3 \mathrm{~d}-\mathrm{f}$ ) and $\gamma 1$ (Figs. 1d, 3c) chains and also nidogen 1 (Figs. 1j, k, 3e) and nidogen 2 (Figs. 1m, n, 3d) and perlecan (Figs. 1b, 3a). Focimatrix was not observed in all antral follicles (Table 3).

The focimatrix in some preantral follicles contained collagen type XVIII (Figs. 1g, h, 3d) and, surprisingly, even in follicles where the follicular basal lamina did not contain collagen type XVIII. However, focimatrix with collagen type XVIII was more abundant in follicles that were surrounded by a basal lamina positive for collagen type XVIII. In co-localisation studies, laminin $\alpha 1$ co-localised with laminin $\gamma 1$ (Figs. 1d, 3c), nidogen 1 (Fig. 3e) and nidogen 2 (Figs. 1m, n, 3f) co-localised with aggregates of laminin $\beta 1$ focimatrix, and laminin $\beta 1$ co-localised with collagen type XVIII (Fig. 1g, h, 3d).

Thecal matrix in antral follicles contained laminin $\beta 1$ (Fig. 1g, h, m, n) and $\gamma 1$ (Fig. 1d, e) chains, perlecan (Fig. 1a-c), nidogen 1 (Fig. 1j, k) and nidogen 2 (Fig. 1m, n) but not collagen type IV $\alpha 1$ and $\alpha 2$ (data not shown), as observed previously (Nakano et al. 2007), laminin $\alpha 1, \alpha 2$, $\alpha 4$ or $\alpha 5$ chains, or collagen type XVIII. Thecal capillary sub-endothelial basal lamina contained the same components as the thecal matrix but additionally collagen type IV $\alpha 1$ and $\alpha 2$ (data not shown) as observed previously (Nakano et al. 2007).

\section{Matrix in atretic follicles}

The follicular basal lamina of atretic follicles showed the same composition as that of healthy follicles. It contained collagen type IV $\alpha 1$ and $\alpha 2$ (as shown previously by Nakano et al. 2007), laminin $\alpha 1$ (Fig. 1f), laminin $\alpha 2$ (Fig. 1c; but only in some large follicles), laminin $\beta 1$ (Fig. 1i, o) and $\gamma 1$ (Fig. 1f) chains, nidogen 1 (Fig. 11) and nidogen 2 (Fig. 1o) and perlecan (Fig. 1c), whereas laminin $\alpha 4$ and $\alpha 5$ and collagen type XVIII (Fig. 1i) were not present. Atretic follicles also possessed focimatrix containing laminin $\alpha 1$ (Fig. 1f), $\beta 1$ (Fig. 1i, o) and $\gamma 1$ (Fig. 1f) chains, nidogens 1 and 2, perlecan (Fig. 1c) and collagen type XVIII (Fig. 1i).

\section{Matrix in corpora lutea}

No parenchymal matrix, as observed in human (Irving-Rodgers et al. 2006b) or bovine (Irving-Rodgers et al. 2004) corpora lutea, was detected and no basal laminas surrounding individual luteal cells were observed. However, luteal capillary sub-endothelial basal laminas were readily ob- served and contained collagen type IV $\alpha 1$ and $\alpha 2$ (data not shown; as observed previously by Nakano et al. 2007), laminin $\alpha 1$ (Fig. 1e), $\beta 1$ (Fig. 1h, m) and $\gamma 1$ (Fig. 1e) chains, nidogen 1 (Fig. 1k) and nidogen 2 (Fig. $1 \mathrm{~m}$ ), perlecan (Fig. 1b) and collagen type XVIII (Fig. 1h) but not laminin $\alpha 2$ (Fig. 1b), $\alpha 4$ or $\alpha 5$ (data not shown) chains. Figure 4 illustrates the co-localisation of some of these ECM components with the endothelial cell marker PECAM-1 (CD31). No differences were observed between luteal capillary sub-endothelial basal lamina composition in corpora lutea from experimental days 5 and 11 (estimated to be days 2 or 9 post-ovulation).

Matrix in other ovary compartments

The epithelial basal lamina of the ovarian surface contained collagen type IV $\alpha 1$ and $\alpha 2$ (data not shown; as observed previously by Nakano et al. 2007), laminin $\beta 1$ (Fig. 1g) and $\gamma 1$ (Fig. 1d, e) chains, nidogen 1 (Fig. 1j, k) and nidogen 2 (Fig. $1 \mathrm{~m}$ ), and perlecan (Fig. 1a, b) but no laminin $\alpha 1$ (Fig. 1d, e), $\alpha 2$ (Fig. 1a, b), $\alpha 4$ or $\alpha 5$ chains or collagen type XVIII (Fig. 1g). Arteriole sub-endothelial basal laminas contained collagen type IV $\alpha 1$ (data not shown). Some of these basal laminas contained laminin $\beta 1$ (Fig. 1g, h), laminin $\gamma 1$ (Fig. 1e-g), nidogen 1 (Fig. 1k, l) and nidogen 2 (Fig. 1m, n), perlecan (Fig. 1b) and collagen type XVIII (Fig. 1g, h), but none contained laminin $\alpha 1, \alpha 2, \alpha 4$ or $\alpha 5$ chains. The basal lamina of arteriole smooth muscle contained collagen type IV $\alpha 1$ and $\alpha 2$; some of these basal laminas contained laminin $\alpha 2$, laminin $\beta 1$, laminin $\gamma 1$ (Fig. 1f), nidogen 1 and 2, perlecan and collagen type XVIII (Fig. 1h) but none contained laminin $\alpha 1, \alpha 4$ or $\alpha 5$.

\section{Discussion}

We provide new information on the localisation of basal lamina components, such as the laminins, nidogens, and the heparan sulphate proteoglycans, perlecan and collagen type XVIII in mouse ovaries, including those associated with the follicular basal lamina, focimatrix, thecal matrix, corpora lutea, vasculature and surface epithelium. Some developmental changes in mouse matrices are common to other species; however, distinct species differences exist, with mouse ovaries clearly having differences in their ECM composition.

The composition of the basal laminas in the follicles of the mouse (Nakano et al. 2007; current study), rat (Frojdman et al. 1998) and bovine (Rodgers et al. 1998; van Wezel et al. 1998; McArthur et al. 2000) have now been examined. As the follicular basal lamina expands in surface area, it changes in composition. In both mouse and bovine, collagen type IV $\alpha 1-\alpha 6$ are present in primordial 
follicles but the expression of collagen type IV $\alpha 3-\alpha 6$ declines during growth, whereas $\alpha 1$ and $\alpha 2$ continue to be expressed (Rodgers et al. 1998; Nakano et al. 2007). However, the stage at which collagen type IV $\alpha 3-\alpha 6$ decline differs between the two species: at the primary stage in mice (Nakano et al. 2007) and at the early antral stage in bovine (Rodgers et al. 1998). This species difference in the precise timing of the loss of collagen type IV $\alpha 3-\alpha 6$ might reflect the finding that mouse follicles reach ovulatory stages much quicker than bovine follicles as they are considerably smaller with fewer granulosa cells at ovulation. Rat follicular basal laminas similarly contain collagen type IV $\alpha 1$ and $\alpha 2$ throughout follicular development (Frojdman et al. 1998). They also have collagen type IV $\alpha 3$ at early stages but exhibit low expression of $\alpha 4$ and $\alpha 5$ from the secondary stage of development (Frojdman et al. 1998).

Laminin $\alpha 1$ is present in the mouse follicular basal lamina at all stages of follicle growth but laminin $\alpha 2$ has only been detected in some follicles, and only at the preantral and antral stages. This same pattern has been observed in the bovine with no apparent explanation of the differences between laminin- $\alpha 2$-positive and laminin- $\alpha 2$ negative follicles (van Wezel et al. 1998), except that it might reflect differences in the theca of these follicles, as laminin $\alpha 2$ is generally of stromal origin (Vuolteenaho et al. 1994; Sorokin et al. 1997; Lefebvre et al. 1999). Laminin $\alpha 4$ or $\alpha 5$ chains have not been detected at any stage in the mouse. Laminin $\beta 1$ and $\gamma 1$ chains are present at all stages of follicular development in the mouse, whereas laminin $\gamma 1$ is also found during follicular growth in bovine but laminin $\beta 1$ only appears transiently during development at the preantral stage (van Wezel et al. 1998). Both nidogen 1 and nidogen 2 have been observed throughout folliculogenesis in the mouse, but they only appear in the bovine at the preantral and antral stages (McArthur et al. 2000; Irving-Rodgers and Rodgers 2006). Of the heparan sulphate proteoglycans, perlecan is present at all stages, whereas collagen type XVIII has been detected only in murine primordial and primary follicles and in some preantral but not in antral follicles. This differs from the bovine in which collagen type XVIII occurs at all stages of follicular development (Irving-Rodgers et al. 2006a; Irving-Rodgers and Rodgers 2006) and perlecan is present from preantral stages onwards (McArthur et al. 2000).

The focimatrix in the mouse clearly differs from that seen in other species. It is found in the preantral and antral follicles of days $0,2,5$ and 11 as larger and fewer aggregates (approximately six in cross sections of a follicle) than those observed in the bovine ovary (Irving-Rodgers et al. 2004) or as fibrous structures throughout the membrana granulosa. Nidogen 1 and 2 and perlecan are fibrous, whereas the aggregates contain collagen type IV $\alpha 1$ and $\alpha 2$, as observed previously (Nakano et al. 2007) and
Fig. 1 Immunlocalisation of matrix components in mouse ovaries. ac Perlecan (green) is localised to the basal lamina of primordial, primary, preantral, antral (a, b) and atretic follicles (c) and in focimatrix (arrows). Laminin $\alpha 2$ (red) can be found in the basal lamina of some preantral, antral $(\mathbf{a}, \mathbf{b})$ and atretic follicles (c). Perlecan is also localised to the sub-endothelial basal lamina of blood vessels in the stroma (star), in the theca (rhombus) and corpora lutea (triangles) and to the basal lamina of smooth muscle cells of arterioles. d-f Laminin $\gamma 1$ (green) is co-localised (yellow) with laminin $\alpha 1$ (red) in the basal lamina of all stages of follicular development (d, e), in atretic (f) and in regressing (open square) follicles. Laminin $\gamma 1$ is also localised to the sub-endothelial basal lamina of blood vessels in the stroma, in the theca (rhombus) and corpora lutea (triangles) and to the basal lamina of smooth muscle cells of arterioles (stars). Laminin $\alpha 1$ is weakly detectable in the corpora lutea. Both laminins are found in the focimatrix (arrows). $\mathbf{g}-\mathbf{i}$ Collagen type XVIII (green) is localised to the basal lamina of primordial, primary and some preantral follicles but not in antral follicles. In comparison, laminin $\beta 1$ (red) is localised in the basal lamina of all follicular stages. Both matrix components are found in the focimatrix (arrows). The sub-endothelial basal lamina in stroma and corpora lutea (triangles) and the basal lamina of smooth muscle cells of arterioles contain collagen XVIII (stars) and some also laminin $\beta 1$. Nuclei are counterstained blue with DAPI (i). j-I Nidogen 1 (green) is expressed in the basal lamina of all follicular stages and in the sub-endothelial basal lamina of blood vessels in the stroma (stars), in the theca (rhombus) and corpora lutea (triangle). It is also detectable in the basal lamina of atretic follicle (l) and in the focimatrix (arrows). m-o Nidogen 2 (green) is co-localised with laminin $\beta 1$ (red) in the basal lamina of all follicular stages $(\mathbf{m}, \mathbf{n})$ and atretic follicles (o). Both markers can be found in the sub-endothelial basal lamina of blood vessels in the stroma (stars), in the theca (rhombus) and corpora lutea (triangles) and in the basal lamina of smooth muscle cells of arterioles. Nuclei are counterstained blue with DAPI (o). Bars $100 \mu \mathrm{m}(\mathbf{a}, \mathbf{b}, \mathbf{d}-\mathbf{h}, \mathbf{j}-\mathbf{n}), 50 \mu \mathrm{m}(\mathbf{c}, \mathbf{i}, \mathbf{o})$

laminins $\alpha 1, \beta 1$ and $\gamma 1$ chains. The focimatrix in some preantral follicles contain collagen type XVIII, including follicles in which the follicular basal lamina is negative for collagen type XVIII. However, focimatrix with collagen type XVIII is more abundant in follicles that have collagen type XVIII in their follicular basal lamina. The focimatrix has not been observed in all antral follicles. Bovine follicles contain all the same components, except that they exhibit laminin $\beta 2$ and not $\beta 1$, but the aggregate is more homogeneous and only appears once follicles are larger than $5 \mathrm{~mm}$ diameter, increasing in amount until ovulation when it is degraded (Irving-Rodgers et al. 2004). The reason that the focimatrix is so variable in mouse ovaries is unknown at this stage.

Throughout the theca interna, basal lamina components have been identified that are not in association with any conventional basal lamina; this has been termed the thecal matrix. In the mouse, the theca matrix contains laminin $\beta 1$ and $\gamma 1$, as in bovine follicles, which contain laminin $\gamma 1$ (van Wezel et al. 1998), but unlike in the bovine, it also contains perlecan and nidogens 1 and 2. Collagen type IV $\alpha 1$ and $\alpha 2$ are also not present in the thecal matrix of the mouse; this is consistent with the previously observation by Nakano et al. (2007) who have found no collagen type IV 


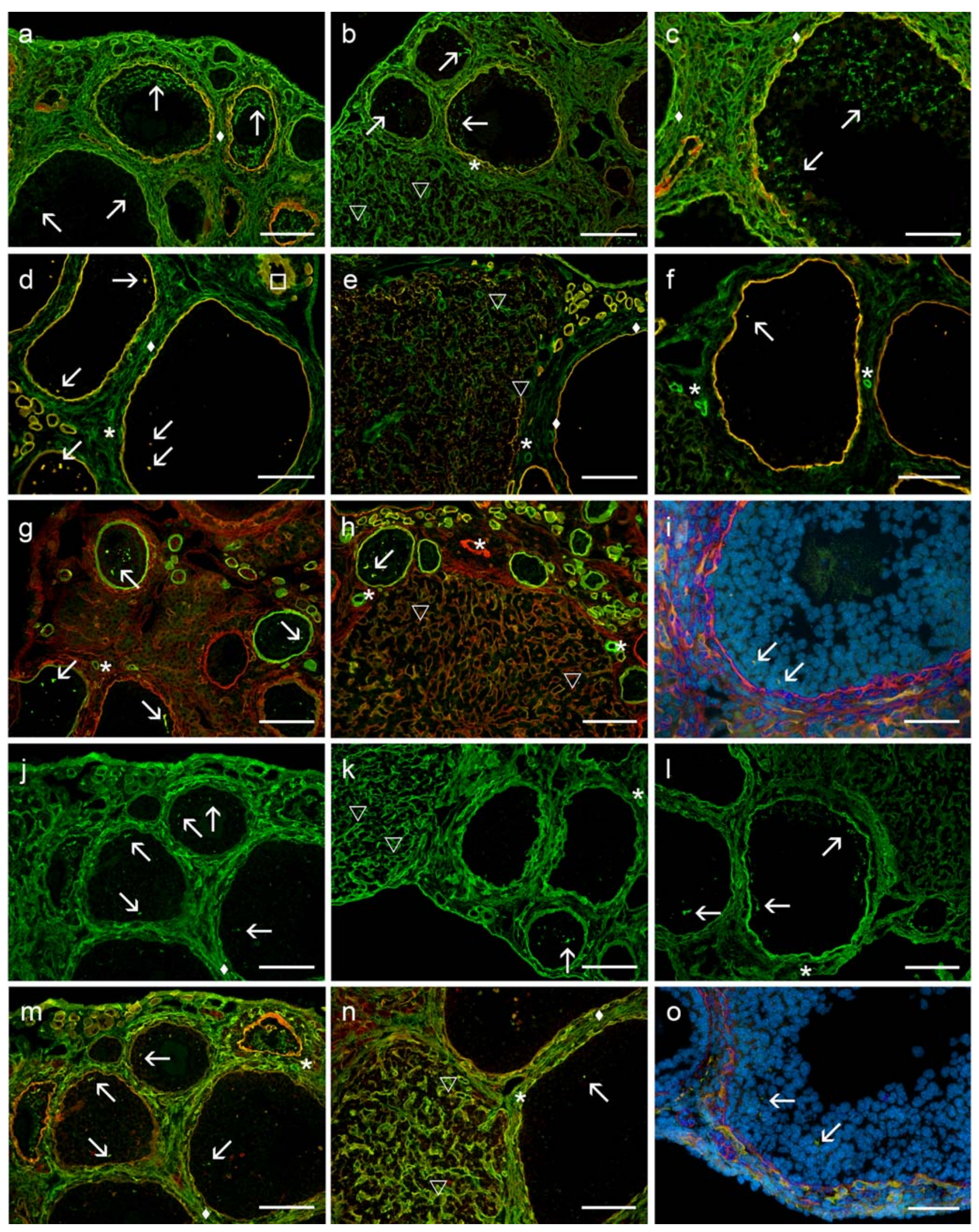


Fig. 2 Immunlocalisation of laminin $\alpha 4$ and $\alpha 5$. Laminin $\alpha 4$ (a, a' counterstained with DAPI; follicles marked with stars) are not localised to structures of the mouse ovary but to the vasculature in mouse heart (c) and skeletal muscle (c'). Laminin $\alpha 5$ (b, b' counterstained with DAPI; follicles marked with stars) is not detectable in mouse ovary, but in the vasculature (stars) and in the basal lamina of the tubule in mouse kidney (d, $G$ glomerulus). Bar $100 \mu \mathrm{m}$ (a-b', d, $50 \mu \mathrm{m}\left(\mathbf{c}, \mathbf{c}^{\prime}\right)$
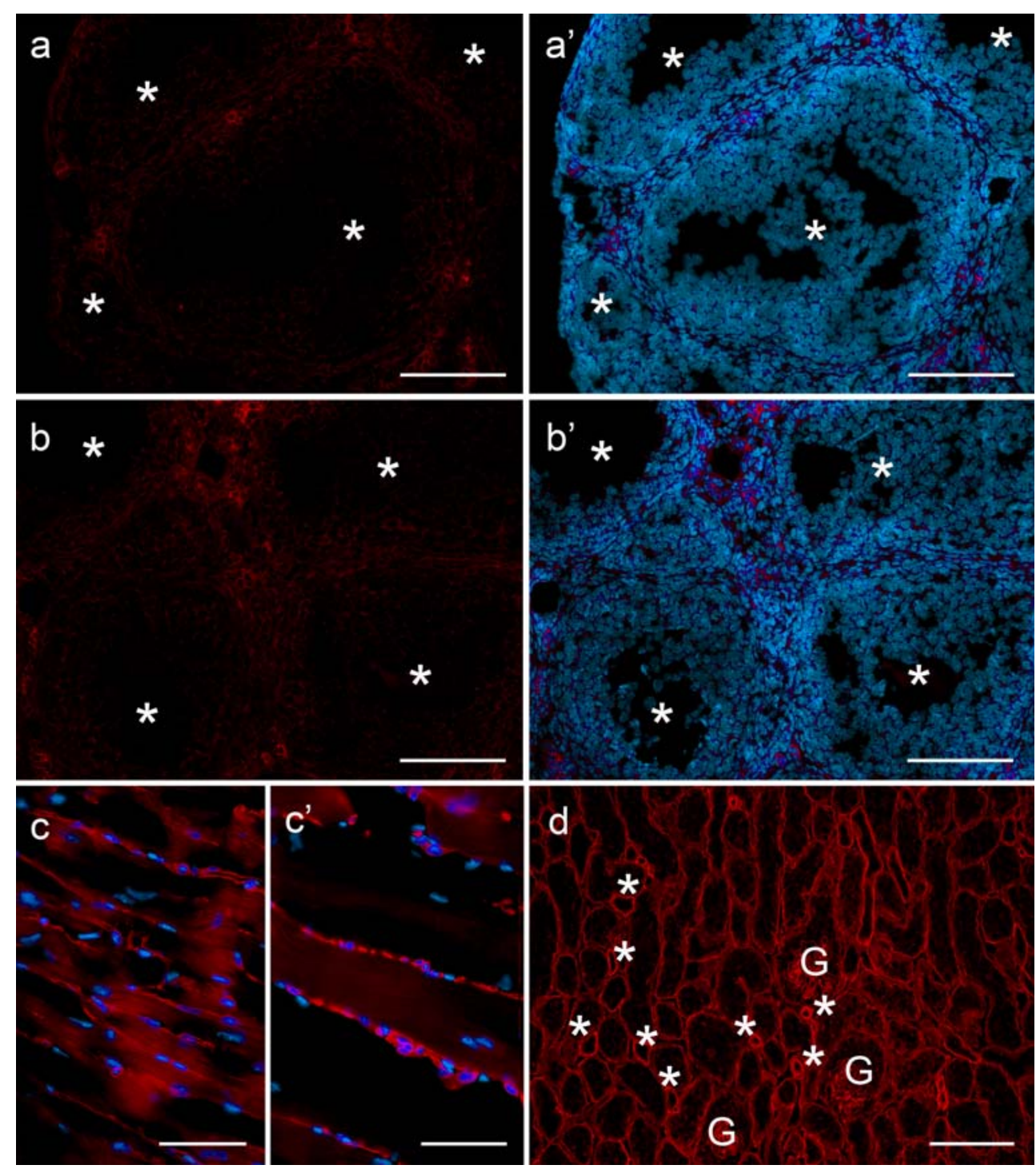

$\alpha 1-\alpha 6$. In mouse, Berkholtz et al. (2006) have illustrated collagen type IV in the theca, although the resolution is not sufficient to distinguish sub-endothelial collagen from thecal matrix.

On comparing bovine and murine follicles, major differences can generally be seen in the expression patterns of nidogens 1 and 2 and perlecan. In the bovine, they appear at the preantral stage and remain present during the antral stage (McArthur et al. 2000; Irving-Rodgers and Rodgers 2006), whereas in mouse, they are present at all stages. In the focimatrix in bovine, these molecules are present as aggregates, just as the collagens and laminins are in mouse, but nidogens 1 and 2 and perlecan have a fibrous pattern within the murine membrana granulosa. The thecal matrix of mice also contains nidogens 1 and 2 and perlecan, which have not been observed in the bovine thecal matrix. Since perlecan can bind nidogens (Hopf et al. 2001), its expression pattern might therefore be similar to those of nidogens 1 and 2 if they are all expressed, and in both species, this is the case. However, the reason that these molecules differ so considerably between mice and bovine is not clear at this stage. We assume that the arrangement of nidogens 1 and 2 and perlecan in mice is the true expression pattern of these components in this species. The more restricted staining pattern in the bovine ovary might be caused by the use of primary antibodies with specificity against mouse, which may react with these components to a more limited extent in the bovine ovary than in the mouse ovary. Alternatively, this could reflect a difference in the cross-linking of these molecules to other less-soluble molecules in the ovary in mice, rather than there being an inherent major difference in the mouse.

The sub-endothelial basal laminas of the mouse thecal capillary contain laminin $\beta 1$ and $\gamma 1$, perlecan, nidogens 1 and 2 and collagen type IV $\alpha 1$ and $\alpha 2$. Collagen type IV $\alpha 1$ and $\alpha 2$ have also been demonstrated in the blood 
Fig. 3 Composition of focimatrix (arrows) in mouse ovaries. a Aggregates and fibres of perlecan (green) in the granulosa layer. The focimatrix does not contain laminin $\alpha 2$ (red). $\mathbf{b}$ Higher magnification of the follicle marked with a star in a. c Co-localisation (yellow) of laminin $\gamma 1$ (green) and laminin $\alpha 1$ (red) in focimatrix. d-f Laminin $\beta 1$ (red) co-localised (yellow) in focimatrix with collagen type XVIII (green) in d, with nidogen 1 (green) in e and with nidogen 2 (green) in f. Bar $50 \mu \mathrm{m}(\mathbf{a}, \mathbf{c}, \mathbf{d}), 25 \mu \mathrm{m}(\mathbf{b}, \mathbf{e}, \mathbf{f})$
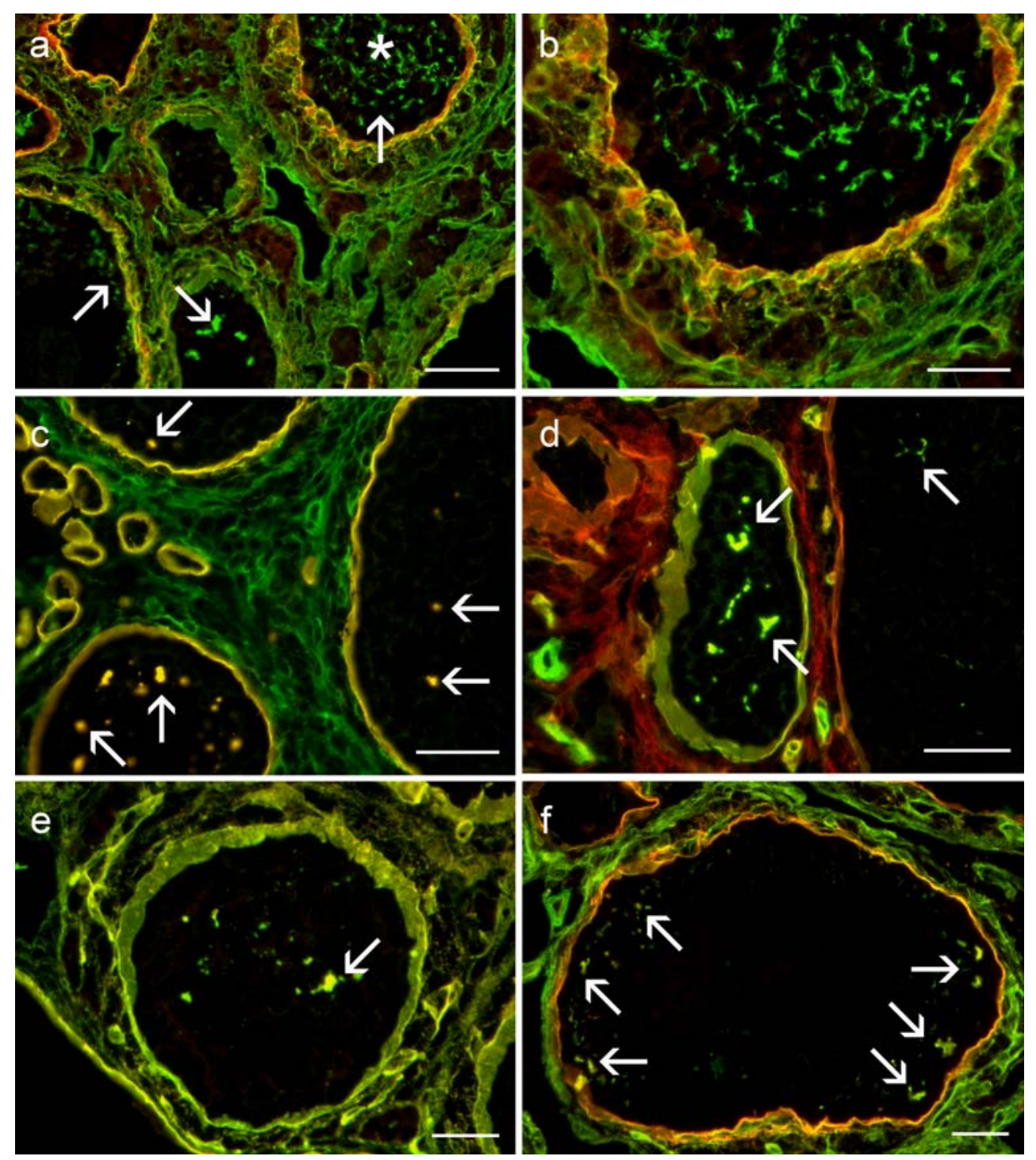

vessels in the theca of rat (Frojdman et al. 1998) and cow, in addition to laminin $\beta 1$ and $\beta 2$ (Rodgers et al. 1998; van Wezel et al. 1998). On interpreting the results from Huet et al. (1997), the sub-endothelial basal lamina of the ovine thecal capillary clearly contains laminin, collagen type IV and a heparan sulphate proteoglycan (Huet et al. 1997).

Basal lamina matrix components have now been identified in the corpora lutea of bovine (Irving-Rodgers et al. 2004), human (Irving-Rodgers et al. 2006b) and murine (Nakano et al. 2007; present results) ovaries. In general, two different types of basal lamina matrix occur in the corpora lutea: sub-endothelial basal laminas and an interstitial matrix located as aggregates at irregular intervals between the non-vascular cells (Irving-Rodgers et al. 2004, 2006b). The earlier speculation about the presence of a basal lamina associated with luteal cells (Deane et al. 1966;
Table 3 Number of preantral and antral follicles examined and mean percentage \pm SEM of follicles with focimatrix identified by immunostaining of cross sections for various extracellular matrix molecules

\begin{tabular}{lll}
\hline Matrix molecule & Number of examined follicles & Percentage of positive follicles \\
\hline Laminin $\alpha 1$ & 94 & $70.1 \pm 7.6$ \\
Laminin $\beta 1$ & 260 & $40.8 \pm 6.8$ \\
Laminin $\gamma 1$ & 94 & $71.5 \pm 9.7$ \\
Nidogen-1 & 260 & $58.3 \pm 5.9$ \\
Nidogen-2 & 120 & $36.7 \pm 7.0$ \\
Perlecan & 230 & $38.7 \pm 2.8$ \\
Collagen type XVIII & 267 & $23.8 \pm 3.9$ \\
\hline
\end{tabular}



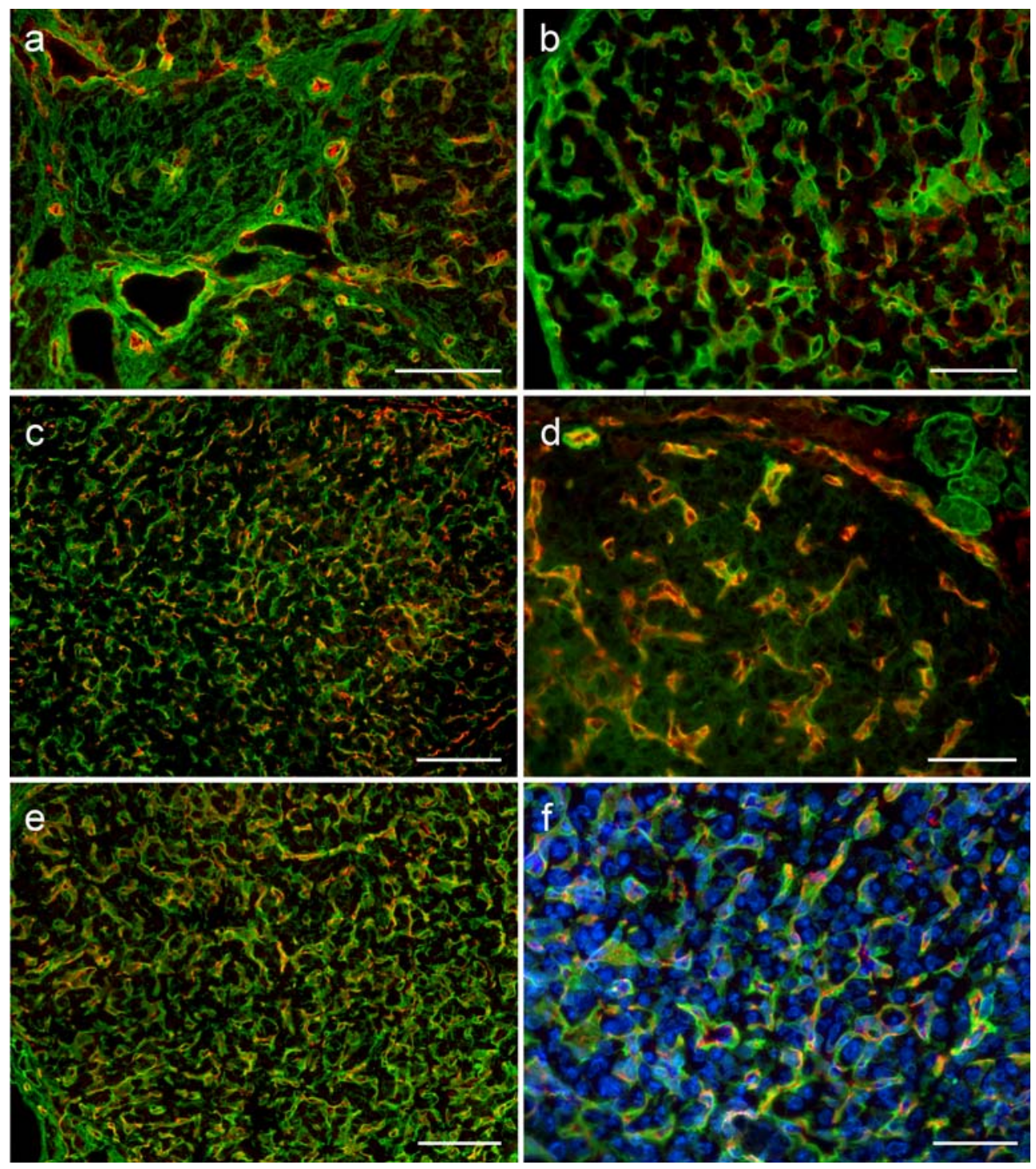

Fig. 4 Co-localisation of matrix components (green) with the endothelial cell marker PECAM-1 (red) in capillary sub-endothelial basal lamina in corpora lutea. a, b Perlecan. $\mathbf{c}$ Laminin $\gamma 1$. d Collagen

Farin et al. 1986; O'Shea 1987; Kenny et al. 1989) has previously been discussed in detail (Irving-Rodgers et al. 2006c). The current study adds further evidence that no such basal lamina exists. In the mouse basal lamina, matrix components are all associated with the sub-endothelial basal laminas, which are positive for collagen type IV $\alpha 1$ and $\alpha 2$, as observed previously (Nakano et al. 2007), and laminin $\alpha 1$, $\beta 1$ and $\gamma 1$ chains, nidogens 1 and 2, perlecan and collagen type XVIII but not laminin $\alpha 2, \alpha 4$ or $\alpha 5$. In addition, no differences have been observed between the younger cyclic corpora lutea and the older pregnancy corpora lutea. The human luteal sub-endothelial basal laminas contain collagen type IV $\alpha 1$ and laminins $\alpha 5, \beta 2$ and $\gamma 1$ (Irving-Rodgers et al. 2006b). In the human, laminin $\alpha 4$ and $\beta 1$ chains occur in the sub-endothelial basal lamina only at mid-luteal to type XVIII. e Nidogen 1. f Nidogen 2. Nuclei are counterstained blue with DAPI (f). Bar $100 \mu \mathrm{m}(\mathbf{a}, \mathbf{c}, \mathbf{e}, \mathbf{f}), 50 \mu \mathrm{m}(\mathbf{b}, \mathbf{d})$

regressing stages and no laminin $\alpha 1$ and $\alpha 3$ chains are present (Irving-Rodgers et al. 2006b).

Endostatin is a proteolytic fragment of collagen type XVIII that can potently inhibit angiogenesis and induces tumour regression in mice (Boehm et al. 1997) and human (Herbst 2002). The presence of collagen type XVIII in the follicular basal lamina early in follicular development but not at the later antral stages correlates inversely with vascularisation of the thecal layers, suggesting that endostatin is produced or released from the follicular basal lamina during follicular development. However, collagen type XVIII null mice are fertile thereby suggesting that collagen type XVIII is not absolutely necessary for follicular development.

Thus, in summary, whereas the structure and composition of basal lamina matrices in mouse is generally similar 
to those in other species, the patterns of nidogens 1 and 2 and perlecan are different in the follicular basal lamina, focimatrix and thecal matrix. This may reflect a difference in the cross-linking of these molecules to other less-soluble molecules in the ovary in mice, rather than representing an inherent major difference in the mouse.

Acknowledgements We are grateful to Drs. M. Dziadek and T. Sasaki for their generous donation of antibodies, to Nicholas Hatzirodos, Leigh Clark and Katie Fraser for assistance with the conduct of these experiments and to Kelly Wicks, Brigit Hines and the IMVS Animal Facility staff for their kind help in animal handling and maintenance.

Open Access This article is distributed under the terms of the Creative Commons Attribution Noncommercial License which permits any noncommercial use, distribution, and reproduction in any medium, provided the original author(s) and source are credited.

\section{References}

Alexopoulos E, Shahid J, Ongley HZ, Richardson MC (2000) Luteinized human granulosa cells are associated with endogenous basement membrane-like components in culture. Mol Hum Reprod 6:324-330

Aumailley M, Bruckner-Tuderman L, Carter WG, Deutzmann R, Edgar D, Ekblom P, Engel J, Engvall E, Hohenester E, Jones JC, Kleinman HK, Marinkovich MP, Martin GR, Mayer U, Meneguzzi G, Miner JH, Miyazaki K, Patarroyo M, Paulsson M, Quaranta V, Sanes JR, Sasaki T, Sekiguchi K, Sorokin LM, Talts JF, Tryggvason K, Uitto J, Virtanen I, Mark K von der, Wewer UM, Yamada Y, Yurchenco PD (2005) A simplified laminin nomenclature. Matrix Biol 24:326-332

Berkholtz CB, Lai BE, Woodruff TK, Shea LD (2006) Distribution of extracellular matrix proteins type I collagen, type IV collagen, fibronectin, and laminin in mouse folliculogenesis. Histochem Cell Biol 126:583-592

Boehm T, Folkman J, Browder T, O'Reilly MS (1997) Antiangiogenic therapy of experimental cancer does not induce acquired drug resistance.Nature 390:404-407

Camaioni A, Salustri A, Yanagishita M, Hascall VC (1996) Proteoglycans and proteins in the extracellular matrix of mouse cumulus celloocyte complexes. Arch Biochem Biophys 325:190-198

Deane HW, Hay MF, Moor RM, Rowson LE, Short RV (1966) The corpus luteum of the sheep: relationships between morphology and function during the oestrous cycle. Acta Endocrinol (Copenh) $51: 245-263$

Dunning KR, Lane M, Brown HM, Yeo C, Robker RL, Russell DL (2007) Altered composition of the cumulus-oocyte complex matrix during in vitro maturation of oocytes. Hum Reprod 22:2842-2850

Durbeej M, Fecker L, Hjalt T, Zhang HY, Salmivirta K, Klein G, Timpl R, Sorokin L, Ebendal T, Ekblom P, Ekblom M (1996) Expression of laminin alpha 1, alpha 5 and beta 2 chains during embryogenesis of the kidney and vasculature. Matrix Biol 15:397-413

Dziadek M, Fujiwara S, Paulsson M, Timpl R (1985a) Immunological characterization of basement membrane types of heparan sulfate proteoglycan. EMBO J 4:905-912

Dziadek M, Paulsson M, Timpl R (1985b) Identification and interaction repertoire of large forms of the basement membrane protein nidogen. EMBO J 4:2513-2518

Erickson AC, Couchman JR (2001) Basement membrane and interstitial proteoglycans produced by MDCK cells correspond to those expressed in the kidney cortex. Matrix Biol 19:769-778
Farin CE, Moeller CL, Sawyer HR, Gamboni F, Niswender GD (1986) Morphometric analysis of cell types in the ovine corpus luteum throughout the estrous cycle. Biol Reprod 35:1299-1308

Frojdman K, Pelliniemi LJ, Virtanen I (1998) Differential distribution of type IV collagen chains in the developing rat testis and ovary. Differentiation 63:125-130

Hay E (1991) Cell biology of extracellular matrix. Plenum, New York Herbst RS (2002)Targeted therapy using novel agents in the treatment of non-small-cell lung cancer.Clin Lung Cancer 3 Suppl 1:S30-S38

Hopf M, Gohring W, Mann K, Timpl R (2001) Mapping of binding sites for nidogens, fibulin-2, fibronectin and heparin to different IG modules of perlecan. J Mol Biol 311:529-541

Huet C, Monget P, Pisselet C, Monniaux D (1997) Changes in extracellular matrix components and steroidogenic enzymes during growth and atresia of antral ovarian follicles in the sheep. Biol Reprod 56:1025-1034

Irving-Rodgers HF, Rodgers RJ (2000) Ultrastructure of the basal lamina of bovine ovarian follicles and its relationship to the membrana granulosa. J Reprod Fertil 118:221-228

Irving-Rodgers HF, Rodgers RJ (2006) Extracellular matrix of the developing ovarian follicle. Semin Reprod Med 24:195-203

Irving-Rodgers HF, Bathgate RA, Ivell R, Domagalski R, Rodgers RJ (2002) Dynamic changes in the expression of relaxin-like factor (INSL3), cholesterol side-chain cleavage cytochrome p450, and 3beta-hydroxysteroid dehydrogenase in bovine ovarian follicles during growth and atresia. Biol Reprod 66:934-943

Irving-Rodgers HF, Harland ML, Rodgers RJ (2004) A novel basal lamina matrix of the stratified epithelium of the ovarian follicle. Matrix Biol 23:207-217

Irving-Rodgers HF, Catanzariti KD, Aspden WJ, D'Occhio MJ, Rodgers RJ (2006a) Remodeling of extracellular matrix at ovulation of the bovine ovarian follicle. Mol Reprod Dev 73:1292-1302

Irving-Rodgers HF, Friden BE, Morris SE, Mason HD, Brannstrom M, Sekiguchi K, Sanzen N, Sorokin LM, Sado Y, Ninomiya Y, Rodgers RJ (2006b) Extracellular matrix of the human cyclic corpus luteum. Mol Hum Reprod 12:525-534

Irving-Rodgers HF, Roger J, Luck MR, Rodgers RJ (2006c) Extracellular matrix of the corpus luteum. Semin Reprod Med $24: 242-250$

Irving-Rodgers HF, Harland ML, Sullivan TR, Rodgers RJ (2009a) Studies of granulosa cell maturation in dominant and subordinate bovine follicles: novel extracellular matrix focimatrix is coordinately regulated with cholesterol side-chain cleavage CYP11A1. Reproduction 137:825-834

Irving-Rodgers HF, Morris S, Collett RA, Peura TT, Davy M, Thompson JG, Mason HD, Rodgers RJ (2009b) Phenotypes of the ovarian follicular basal lamina predict developmental competence of oocytes. Hum Reprod 24:936-944

Kenny N, Farin CE, Niswender GD (1989) Morphometric quantification of mitochondria in the two steroidogenic ovine luteal cell types. Biol Reprod 40:191-196

Kreeger PK, Fernandes NN, Woodruff TK, Shea LD (2005) Regulation of mouse follicle development by follicle-stimulating hormone in a three-dimensional in vitro culture system is dependent on follicle stage and dose. Biol Reprod 73:942-950

Lefebvre O, Sorokin L, Kedinger M, Simon-Assmann P (1999) Developmental expression and cellular origin of the laminin alpha2, alpha4, and alpha5 chains in the intestine. Dev Biol 210:135-150

Marneros AG, Olsen BR (2005) Physiological role of collagen XVIII and endostatin. FASEB J 19:716-728

McArthur ME, Irving-Rodgers HF, Byers S, Rodgers RJ (2000) Identification and immunolocalization of decorin, versican, perlecan, nidogen, and chondroitin sulfate proteoglycans in bovine small-antral ovarian follicles. Biol Reprod 63:913-924

Nakano K, Naito I, Momota R, Sado Y, Hasegawa H, Ninomiya Y, Ohtsuka A (2007) The distribution of type IV collagen alpha 
chains in the mouse ovary and its correlation with follicular development. Arch Histol Cytol 70:243-253

O'Shea JD (1987) Heterogeneous cell types in the corpus luteum of sheep, goats and cattle. J Reprod Fertil Suppl 34:71-85

Papaccio G, Latronico MV, Pisanti FA, Federlin K, Linn T (1998) Adhesion molecules and microvascular changes in the nonobese diabetic (NOD) mouse pancreas. An NO-inhibitor (L-NAME) is unable to block adhesion inflammation-induced activation. Autoimmunity 27:65-77

Papathanasiou P, Goodnow CC (2005) Connecting mammalian genome with phenome by ENU mouse mutagenesis: gene combinations specifying the immune system. Annu Rev Genet 39:241-262

Paulsson M (1992) Basement membrane proteins: structure, assembly, and cellular interactions. Crit Rev Biochem Mol Biol 27:93-127

Pearsall N, Bhattacharya G, Wisecarver J, Adams J, Cosgrove D, Kimberling W (2002) Usherin expression is highly conserved in mouse and human tissues. Hear Res 174:55-63

Rodgers HF, Irvine CM, Wezel IL van, Lavranos TC, Luck MR, Sado Y, Ninomiya Y, Rodgers RJ (1998) Distribution of the alpha1 to alpha6 chains of type IV collagen in bovine follicles. Biol Reprod 59:1334-1341

Rodgers RJ, Irving-Rodgers HF, Russell DL (2003) Extracellular matrix of the developing ovarian follicle. Reproduction 126:415-424

Sado Y, Kagawa M, Kishiro Y, Sugihara K, Naito I, Seyer JM, Sugimoto M, Oohashi T, Ninomiya Y (1995) Establishment by the rat lymph node method of epitope-defined monoclonal antibodies recognizing the six different alpha chains of human type IV collagen. Histochem Cell Biol 104:267-275

Sado Y, Kagawa M, Naito I, Ueki Y, Seki T, Momota R, Oohashi T, Ninomiya Y (1998) Organization and expression of basement membrane collagen IV genes and their roles in human disorders. J Biochem (Tokyo) 123:767-776

Schymeinsky J, Nedbal S, Miosge N, Poschl E, Rao C, Beier DR, Skarnes WC, Timpl R, Bader BL (2002) Gene structure and functional analysis of the mouse nidogen- 2 gene: nidogen- 2 is not essential for basement membrane formation in mice. Mol Cell Biol 22:6820-6830

Sixt M, Engelhardt B, Pausch F, Hallmann R, Wendler O, Sorokin LM (2001) Endothelial cell laminin isoforms, laminins 8 and 10, play decisive roles in $\mathrm{T}$ cell recruitment across the blood-brain barrier in experimental autoimmune encephalomyelitis. J Cell Biol 153:933-946

Sorokin LM, Pausch F, Durbeej M, Ekblom P (1997) Differential expression of five laminin alpha (1-5) chains in developing and adult mouse kidney. Dev Dyn 210:446-462

Sorokin LM, Maley MA, Moch H, Mark H von der, Mark K von der, Cadalbert L, Karosi S, Davies MJ, McGeachie JK, Grounds MD (2000) Laminin alpha4 and integrin alpha6 are upregulated in regenerating dy/dy skeletal muscle: comparative expression of laminin and integrin isoforms in muscles regenerating after crush injury. Exp Cell Res 256:500-514

Timpl R, Brown JC (1996) Supramolecular assembly of basement membranes. Bioessays 18:123-132

Wezel IL van, Rodgers HF, Rodgers RJ (1998) Differential localization of laminin chains in bovine follicles. J Reprod Fertil 112:267-278

Vuolteenaho R, Nissinen M, Sainio K, Byers M, Eddy R, Hirvonen H, Shows TB, Sariola H, Engvall E, Tryggvason K (1994) Human laminin $\mathrm{M}$ chain (merosin): complete primary structure, chromosomal assignment, and expression of the $\mathrm{M}$ and $\mathrm{A}$ chain in human fetal tissues. J Cell Biol 124:381-394

Xu M, Banc A, Woodruff TK, Shea LD (2009) Secondary follicle growth and oocyte maturation by culture in alginate hydrogel following cryopreservation of the ovary or individual follicles. Biotechnol Bioeng 103:378-386

Yamada S, Fujiwara H, Honda T, Higuchi T, Nakayama T, Inoue T, Maeda M, Fujii S (1999) Human granulosa cells express integrin alpha2 and collagen type IV: possible involvement of collagen type IV in granulosa cell luteinization. Mol Hum Reprod 5: $607-617$ 